\title{
Complex Cystic Breast Masses: An Ultrasound Imaging Review
}

\author{
Niketa Chandrakant Chotai ${ }^{1,2}$, Harold Yim ${ }^{1}$, Elizabeth Chun Mei Fok ${ }^{1}$, Siu Cheng Loke1, \\ Hollie Mei Yeen Lim
}

${ }^{1}$ Department of Diagnostic Radiology, Tan Tock Seng Hospital, Singapore

${ }^{2}$ RadLink Imaging Centre, Singapore

Email: ^niketachotai@gmail.com

How to cite this paper: Chotai, N.C., Yim, H., Fok, E.C.M., Loke, S.C. and Lim, H.M.Y. (2022) Complex Cystic Breast Masses: An Ultrasound Imaging Review. Advances in Breast Cancer Research, 11, 31-49. https://doi.org/10.4236/abcr.2022.111002

Received: November 30, 2021

Accepted: January 8, 2022

Published: January 11, 2022

Copyright $\odot 2022$ by author(s) and Scientific Research Publishing Inc. This work is licensed under the Creative Commons Attribution International License (CC BY 4.0).

http://creativecommons.org/licenses/by/4.0/

\begin{abstract}
Cystic lesions are very commonly encountered entities in the breast. Among these, Complex Cystic Breast Masses (CCBMs), which contain both anechoic and echogenic components, can result in a variety of imaging appearances. These include cystic lesions with thick walls and/or internal septa, intracystic nodular lesions, and solid-cystic masses of varying compositions. Ultrasound is the mainstay for evaluating cystic lesions, and thus recognizing the imaging features appropriately and suggesting suitable interventional procedures are included in their management. In this pictorial essay, we describe the wide range of ultrasound appearances of CCBMs with a number of clinically encountered examples from our institution. This article would enhance the understanding of readers in possible differentials to be included in their clinical practice and to suggest appropriate further intervention, when deemed necessary.
\end{abstract}

\section{Keywords}

Breast, Complex, Cystic Masses, Ultrasound

\section{Introduction}

Cystic lesions are some of the most commonly encountered entities in the breast. They can often be a diagnostic challenge to the radiologist, given their myriad appearances and a wide variety of benign and malignant pathologies. Ultrasound (US) plays an important role in their characterization, categorizing them into simple, complicated or complex cysts, each with different management strategies and prognostic outcomes.

Simple cysts are essentially well-defined anechoic lesions, having impercepti- 
ble walls and increased through-transmission. No intervention is required for these BI-RADS 2 lesions, except for large, painful cysts aspirated for symptomatic relief.

Complicated cysts contain internal echoes, due to cellular debris, blood, proteinaceous material, cholesterol, and other content. While no solid component or internal vascularity is present, differentiating these from a solid lesion may be difficult. These BI-RADS 3 lesions are rarely malignant, with a cancer risk of $<2 \%$ [1]. It is recommended for them to establish stability, with intervention considered if they are symptomatic or growing.

Complex Cystic Breast Masses (CCBMs), containing anechoic as well as echogenic components, result in a variety of imaging appearances. Doshi et al. [1], adapting criteria previously described by Berg et al. [2], classified these into four types (Table 1), based on their sonographic features. Type 1 lesions show a thick outer wall, internal septa, or both. Type 2 masses contain one or more intracystic masses. Type 3 masses have mixed cystic and solid components, and are at least $50 \%$ cystic. Type 4 masses are at least $50 \%$ solid. As interpreting these descriptions of type 2 and 3 masses may result in some overlapping findings in practice, we add a clarification to the definition of type 2 masses, in that the intracystic masses are peripheral and nodular.

This pictorial essay aims to describe many benign, atypical and malignant $b$ reast pathologies presenting as CCBMs (Table 2). We contribute and describe a number of such examples, encountered at our institution over the past decade. The imaging was performed on a variety of ultrasound machines used at our institution including Philips iU22 xMATRIX, Toshiba Aplio 500 and GE LOGIQ E9.

\section{Type 1 Masses}

Fibroadenomatoid hyperplasia

This rare benign lesion, featuring an increased number of intralobular ductules and sclerosis of the intralobular connective tissue, most commonly occurs in younger patients as a focal palpable lesion, or breast pain/tenderness. A

Table 1. Classification of complex cystic breast masses based on morphological criteria.

\begin{tabular}{cc}
\hline & Criteria \\
\hline Type 1 & Thick outer wall (at least $0.5 \mathrm{~mm}$ ) \\
& Thick internal septa (at least $0.5 \mathrm{~mm}$ ) \\
Type 2 & One or more intracystic masses \\
& Masses are peripheral, nodular \\
Type 3 & Mixed cystic and solid components \\
& Predominantly (at least 50\%) cystic \\
& Mixed cystic and solid components \\
Type 4 & Predominantly (at least $50 \%$ ) solid \\
& May include eccentric cystic components
\end{tabular}


Table 2. Summary of lesions based on CCBMs classification, as well as benign, atypical and malignant changes.

\begin{tabular}{|c|c|c|c|}
\hline & Benign & Atypical & Malignant \\
\hline Type 1 & $\begin{array}{l}\text { Fibroadenomatoid } \\
\text { hyperplasia }\end{array}$ & $\begin{array}{c}\text { Flat epithelial } \\
\text { atypia }\end{array}$ & \\
\hline Type 2 & $\begin{array}{l}\text { Fibrocystic change } \\
\text { Apocrine metaplasia } \\
\text { Intraductal papilloma } \\
\text { Intracystic papilloma }\end{array}$ & $\begin{array}{l}\text { Atypical ductal } \\
\text { hyperplasia }\end{array}$ & $\begin{array}{l}\text { Papillary } \\
\text { carcinoma }\end{array}$ \\
\hline Type 3 & $\begin{array}{l}\text { Breast abscess } \\
\text { Fat necrosis } \\
\text { Post-operative seroma } \\
\text { Adenomyoepithelioma }\end{array}$ & & Papillary DCIS \\
\hline Type 4 & $\begin{array}{l}\text { Breast abscess } \\
\text { Granulomatous mastitis } \\
\text { Galactocoele } \\
\text { Lymphangioma } \\
\text { Foreign body granuloma } \\
\text { Myxoid fibroadenoma } \\
\text { Benign phyllodes }\end{array}$ & & $\begin{array}{l}\text { Invasive ductal carcinoma } \\
\text { Invasive mucinous carcinoma } \\
\text { DCIS } \\
\text { Encysted papillary carcinoma } \\
\text { Malignant phyllodes } \\
\text { Metaplastic breast carcinoma } \\
\text { Angiosarcoma }\end{array}$ \\
\hline
\end{tabular}

*DCIS: ductal carcinoma in situ.

circumscribed, heterogeneously hypoechoic mass may be seen, with internal echogenic septations and vascularity [3] (Figure 1).

Flat epithelial atypia

This refers to an alteration of native intraductal epithelial cells, replaced by cuboidal/columnar cells with mild cytologic atypia. Absence of architectural atypia distinguishes this from Atypical Ductal Hyperplasia (ADH) and Ductal Carcinoma In Situ (DCIS), and may present as an irregular microlobulated lesion, with hypoechoic or complex cystic features [4] (Figure 2).

\section{Type 2 Masses}

Fibrocystic change

This is a benign change in the Terminal Ductal Lobular Unit (TDLU), sometimes with fibrosis. It presents with a cyclic pattern of recurring and remitting pain and tenderness, tied to the menstrual cycle, with diffuse or regional areas of firmness. There may be prominent fibroglandular tissue with progressively coarsened echotexture from cumulative fibrotic changes, along with small cysts, sometimes clustered [5] (Figure 3).

\section{Apocrine metaplasia}

This benign epithelial alteration in the lobular portion of the TDLU is associated with fibrocystic change. Secretory columnar cells replace the normal cuboidal epithelium, and the resulting apocrine secretions form microcysts. As these gradually increase in size, several adjacent acini fuse to form larger cystic 


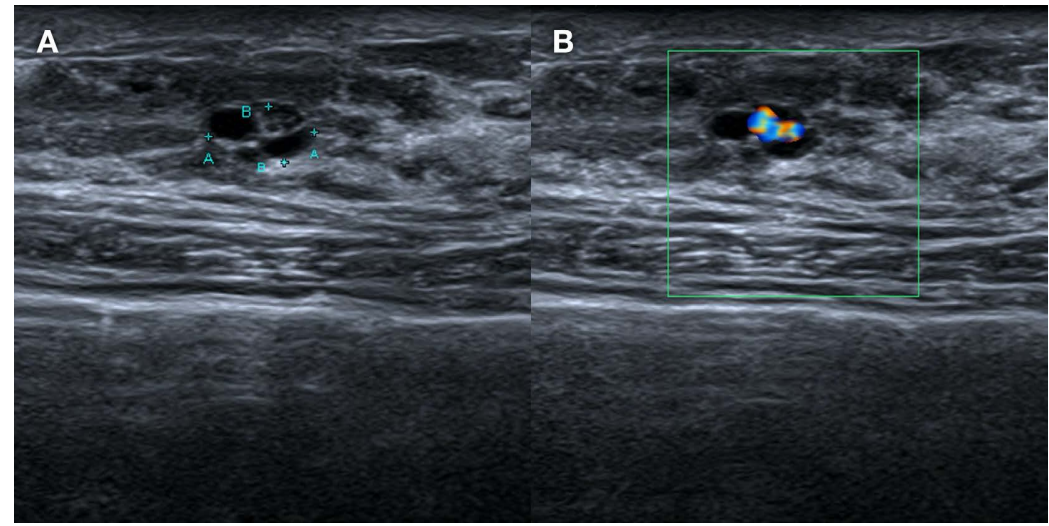

Figure 1. A 41-year-old female presented with breast pain. (A) Sonographic images show a type $1 \mathrm{CCBM}$ at the 6 o'clock subareolar position-a cyst with multiple septa; (B) Internal vascularity is also noted in the corresponding sonographic Doppler images. Histology was reported as fibroadenomatoid hyperplasia.

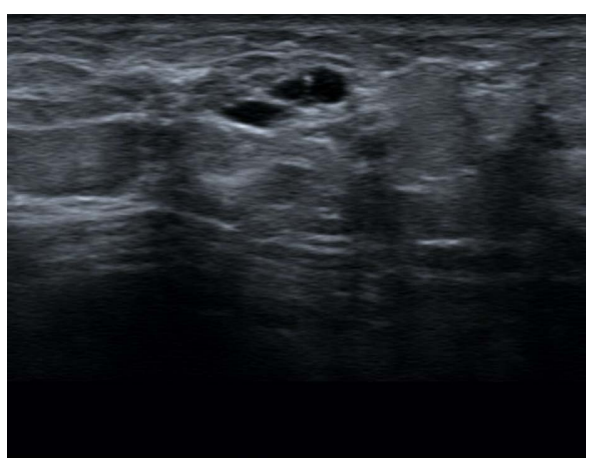

Figure 2. A 58-year-old female was found to have a small cluster of microcalcifications in the lower inner quadrant of the left breast on screening mammogram (not shown). Corresponding sonographic image over the 7 o'clock region shows a type 1 CCBM-a cystic lesion with a thickened septum. Results of an US-guided core biopsy revealed FEA. No DCIS was reported on excision biopsy of this lesion.

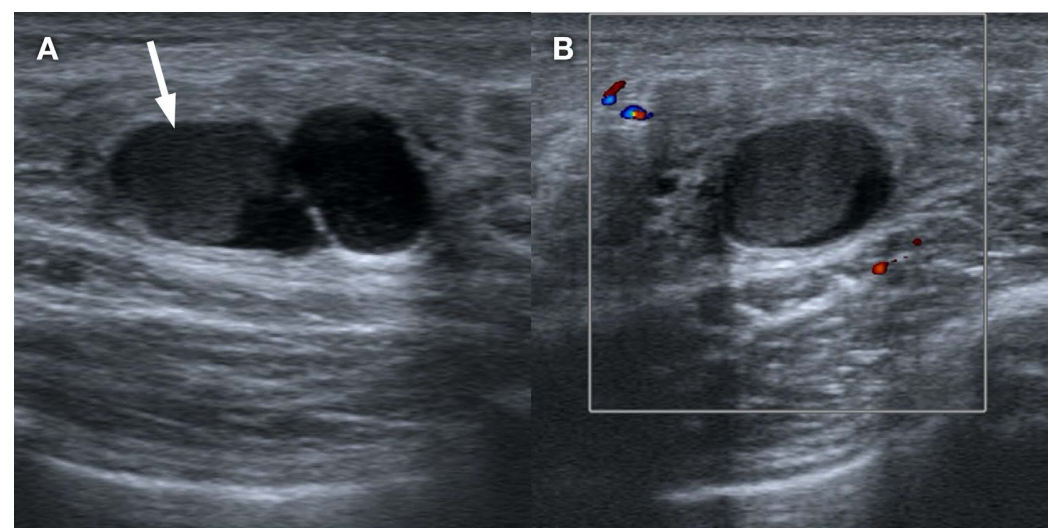

Figure 3. A 31-year-old female presented with mastalgia in the left breast. (A) Sonographic images show a lesion in the left breast 2 o'clock location with type 2 CCBM features-a thinly-septated cyst with a non-dependent, echogenic and intracystic nodular component (arrow); (B) No internal vascularity is demonstrated on color Doppler. This being the area of concern, biopsy was performed on patient's request and was reported as fibrocystic change. 
spaces [6]. Typically, clustered small anechoic foci with intervening septations and posterior acoustic enhancement are seen (Figure 4).

Intraductal papilloma

These are the most common mass lesions in the major lactiferous ducts, usually presenting in the late reproductive or menopausal years, with serous or serosanguinous discharge. They appear as well-defined solid vascularized stalks or masses in the subareolar region (Figure 5), frequently within dilated ducts. Multiple lesions occur in $10 \%$ of cases, more common in younger patients, and confer an increased risk of malignancy [7].

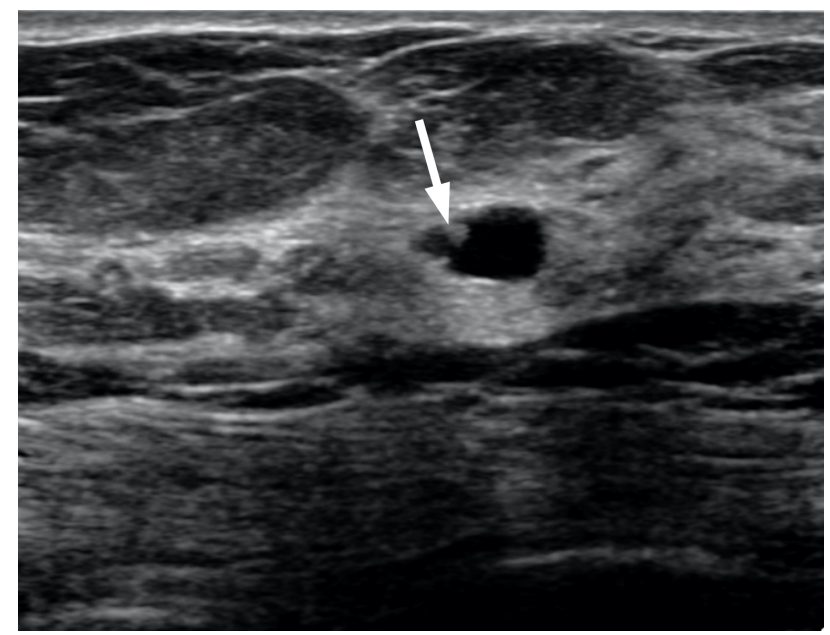

Figure 4. A 58-year-old female was referred for biopsy for abnormal findings detected on screening study. Mammogram (not shown) showed a circumscribed isodense mass with lobulated margins. Sonographic image shows a type 2 CCBM in the left breast-a cystic lesion with a tiny, eccentric mural nodule noted within (arrow). In view of positive family history, biopsy was recommended and histology was reported as apocrine metaplasia.

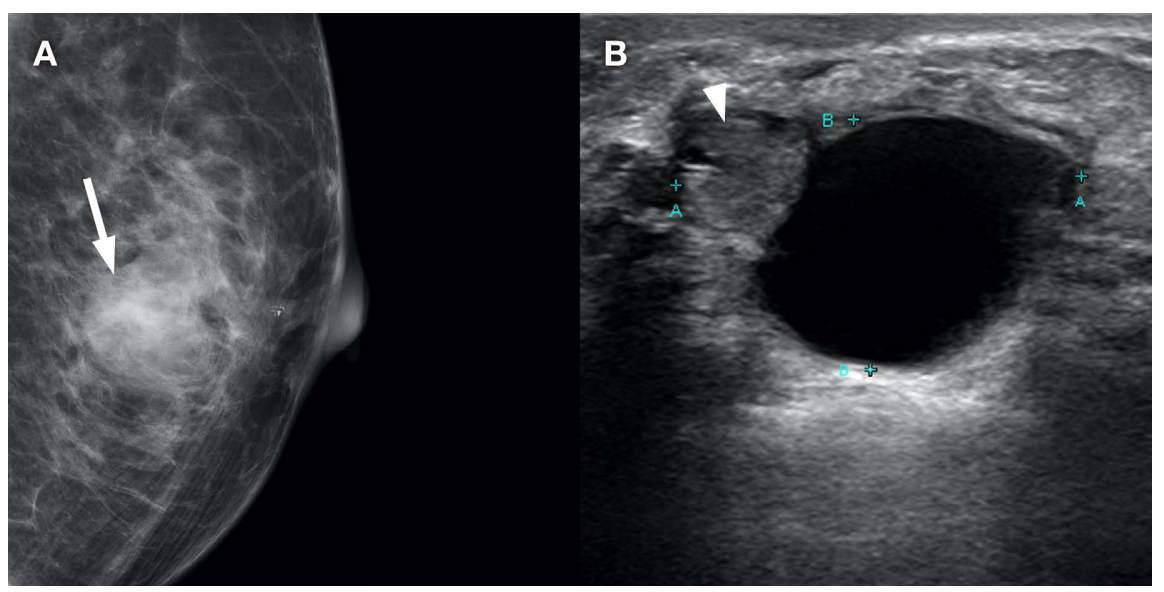

Figure 5. A 69-year-old female presented with palpable lump in the left breast. (A) Mammographic image shows a partially-circumscribed high-density mass in the left retroareolar region (arrow); (B) Corresponding sonographic image shows a type 2 CCBM with a small, nodular solid component located eccentrically in a large cystic mass (arrow). Ultrasound guided biopsy from the solid component of the lesion was reported as intraductal papilloma. 


\section{Intracystic papilloma}

As an uncommon manifestation of intraductal papilloma, this is comprised of a branching central fibrovascular core covered by epithelium, and extends out from the ductal wall into the lumen. The duct becomes obstructed and dilated as the papilloma continues to grow. The resulting cystic lesion is usually well-circumscribed and septated, with an irregular macrolobulated hypoechoic internal nodule [8] (Figure 6).

Atypical ductal hyperplasia

This refers to an intraductal proliferation of epithelial cells, having some of the histological features of DCIS, thus considered borderline. Groups of indeterminate microcalcifications are the common mammographic feature. Sonographically (Figure 7), it is a mildly hypoechoic, microlobulated mass, associated with a fluid-filled duct [9].

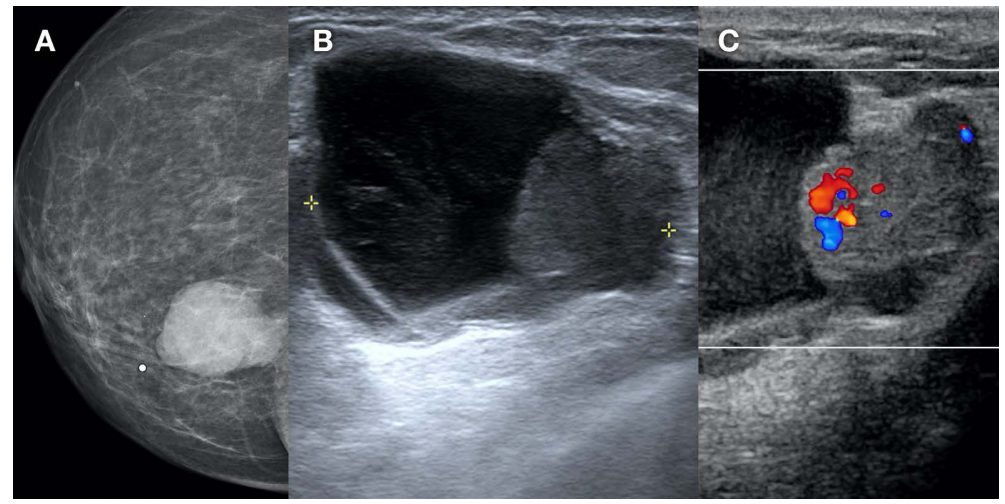

Figure 6. An 81-year-old female presented with palpable right breast lump. (A) Mammographic image shows an oval, circumscribed, high-density mass underneath the palpable region of concern; (B) Sonographic image shows a type 2 CCBM in the 4 o'clock region; (C) Color Doppler image demonstrates a vascularized solid component located peripherally. Histology performed from the solid component, under ultrasound guidance, was reported as intracystic papilloma.

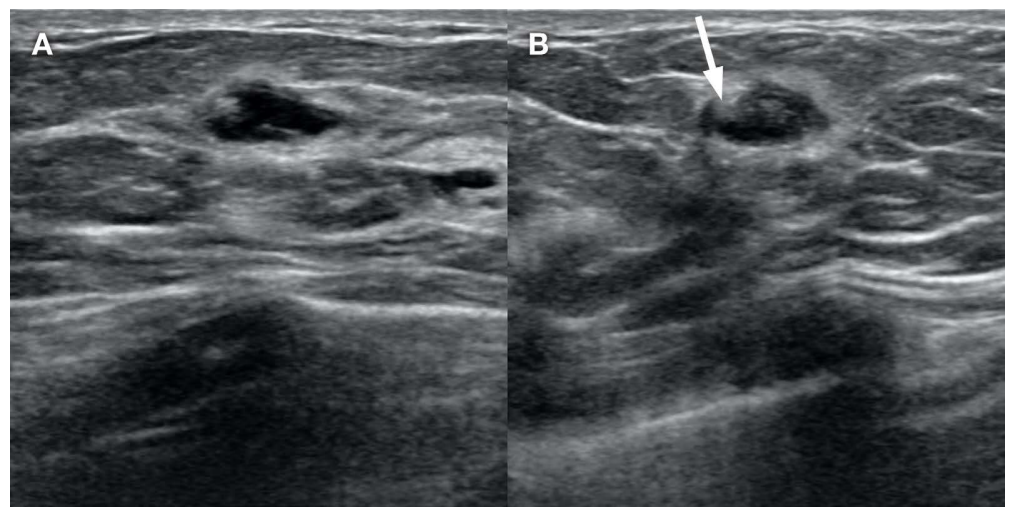

Figure 7. A 48-year-old female presented with a palpable lump in the left breast. Mammography revealed dense breast parenchyma with no focal abnormality (not shown). (A) and (B) Diagnostic sonographic images show a predominantly cystic lesion with a small peripheral nodule in the 7 o'clock position (arrow) correlating with the clinically palpable lump. Biopsy performed from the solid component of this type 2 CCBM revealed ADH. 


\section{Papillary carcinoma}

Comprising $1 \%-2 \%$ of breast malignancies, these may manifest most commonly in post-menopausal women as palpable retroareolar masses or nipple discharge. They can present as a hypoechoic solid mass, or complex cyst with septations or mural-based nodularity. Anechoic regions represent cystic components or haemorrhage [8] (Figure 8).

\section{Type 3 Masses}

\section{Breast abscess}

Typically with these, there is antecedent mastitis, though certain underlying conditions such as diabetes or rheumatoid arthritis may be contributory. Irregular, thick-walled and multi-loculated collections with debris may be demonstrated (Figure 9 and Figure 10). Overlying skin thickening and peripheral vascularity are common. Ultrasound plays an important role in guiding aspiration, helping to relieve symptoms and enhancing response to antibiotic therapy.

\section{Fat necrosis}

Localized inflammation causes saponification and necrosis of fatty tissue, due to causes such as trauma, post-surgery, or radiation therapy, resulting in oily fluid and cellular debris. During the reparative phase, encapsulation by fibrosis occurs, sometimes with wall calcification. Appearances vary depending on the degree of fibrosis, but usually show well-defined anechoic or hypoechoic lesions, with mural nodules or echogenic bands [10] (Figure 11). Correlation with mammography is recommended to confirm the presence of fat, helping avoid unnecessary biopsy.

\section{Post-operative seroma}

Post-surgical collection of serous fluid can occur as an early complication,



Figure 8. A 77-year-old female presented with a palpable right breast lump. (A) Mammographic image of the right breast shows a large, lobulated high-density lesion in the retroareolar region; (B) Sonographic image shows a large type 2 CCBM in the retroareolar breast, with a nodular eccentric solid component. Internal vascularity is also noted in the solid component. Biopsy performed from the solid component of this type 2 CCBM revealed papillary carcinoma. 




Figure 9. A 39-year-old female presented with painful right breast lump. Sonographic Doppler image shows a type 3 CCBM in the subareolar region, predominantly cystic with thick, irregular wall and peripheral vascularity. The patient underwent US-guided aspiration and core needle biopsy of the lesion wall, which revealed findings consistent with an abscess.

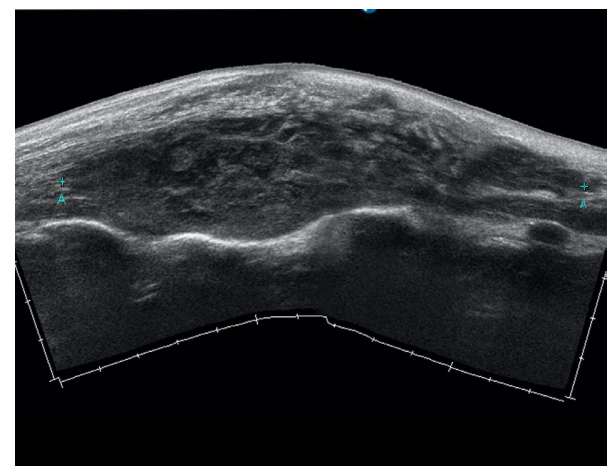

Figure 10. A 61-year-old female presented with right breast inflammatory changes, with overlying skin redness, warmth and tenderness. Sonographic image shows a large type 4 CCBM. $20 \mathrm{cc}$ of thick purulent material was aspirated under US guidance, followed by core needle biopsy of the non-liquified portion, which confirmed an abscess.



Figure 11. A 53-year-old patient with breast surgery a year prior. Surveillance mammogram in this patient (not shown) demonstrated post-surgical changes with surgical clips and a fat containing lesion in the operative bed. (A) and (B) Corresponding sonographic images show a type 2 CCBM in the region of scar-a cystic lesion with lobulated rounded echoes within (arrow). No biopsy was indicated. 
from potential spaces left following lumpectomy/mastectomy or axillary dissection. These are typically anechoic, and as they mature, result in a thickened and almost nodular appearance of the wall (Figure 12).

Adenomyoepithelioma

These rare tumors feature proliferation of epithelial and myoepithelial elements in the breast lobules and ducts, manifesting clinically as non-tender palpable masses. Most are deemed benign, though if presenting with rapid enlargement, malignant change may be considered. They are microlobulated and hypoechoic, with posterior acoustic enhancement and increased peripheral vascularity (Figure 13). Cystic components, as well as surrounding ductal dilatation, have also been reported [11].



Figure 12. A 58-year-old female who had recent excision of a right breast lump, on follow-up. (A) Mammographic image shows a large, rounded subareolar opacity; (B) Corresponding sonographic image shows a type 3 CCBM, largely anechoic with a slightly hyperechoic, thickened and nodular rim (arrow).

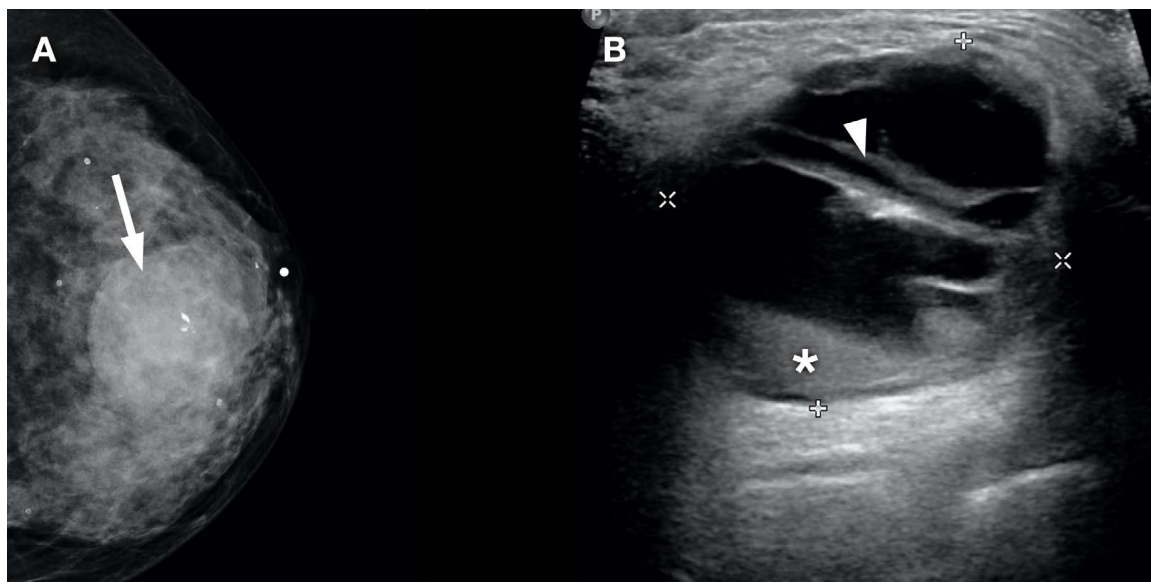

Figure 13. A 64-year-old female presented with a palpable lump in the left breast. (A) Mammogram shows a large, partially-circumscribed mass in the central aspect of the left breast (arrow); (B) Sonographic image shows a type 3 CCBM at the 5 o'clock locationlargely cystic with thickened septa (arrowhead), small solid components adjacent to the septa, and mobile debris (asterisk) in the dependent aspect. Histology was reported as adenomyoepithelioma. 


\section{Papillary DCIS}

As a variant of DCIS, this features prominent papillae with fibrovascular cores, and is frequently seen with other architectural patterns of DCIS. Multiple ducts can be involved, as with all other variants. The regions involved may be sonographically occult, or show ill-defined hypoechoic masses or small calcifications [8] (Figure 14).

\section{Type 4 Masses}

\section{Granulomatous mastitis}

This inflammatory reaction, centered on the lobules, usually affects women of child-bearing age or using oral contraceptives; no specific causative agent has been described. Most commonly presenting with masses, patients may also report pain, erythema, axillary lymphadenopathy, and draining sinus tracts. It appears as an irregular hypoechoic lesion, sometimes with tubular extensions (Figure 15). Associated fat necrosis and abscess formation can result in cystic appearance.

Galactocoele

This develops from ductal obstruction over weeks to months, usually painless in lactating women. The appearance is variable (Figure 16), usually anechoic, but increasingly echogenic based on higher milk/fat content, occasionally with fat-fluid levels. Atypically, they may exhibit microlobulated margins, irregular shape, and posterior shadowing [12], mimicking sinister lesions, requiring multi-modality imaging for further characterization.

Lymphangioma

These are rare entities in the breast and axilla, known to occur in the subareolar region, upper outer quadrant, and tail of Spence [13], consisting of dilated lymph channels lined by endothelium. They are lobulated and anechoic, with variably thick internal septa and internal debris (Figure 17).



Figure 14. A 66-year-old female presented with an enlarging left breast lump. (A) Mammographic image shows an irregular high-density, palpable mass in the upper outer quadrant; (B) Sonographic image shows a large type 3 CCBM in the 2 o'clock location, with a microlobulated solid component seen eccentrically placed. Histology from the solid component was reported as papillary DCIS. 


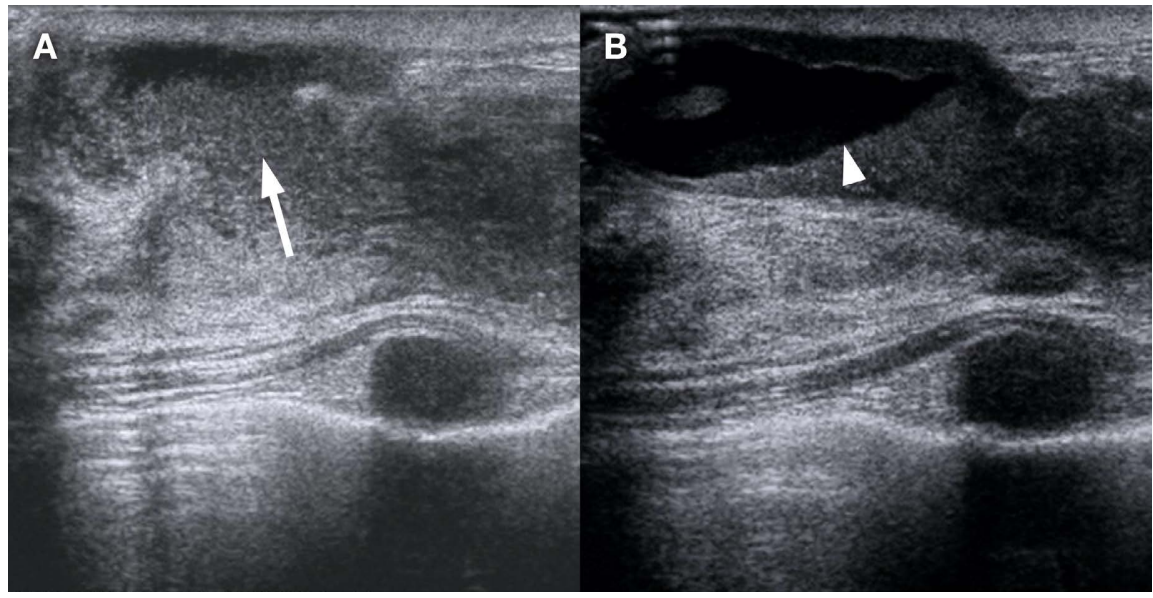

Figure 15. A 33-year-old female presented with a painful right breast lump. Mammography was not performed initially; typically, they feature asymmetric density, with or without overlying skin thickening, as well as axillary lymphadenopathy. (A) Sonographic image shows an ill-defined type 4 CCBM (long arrow), consistent with an abscess (arrow). US-guided aspiration was performed which yielded $7 \mathrm{cc}$ of pus; (B) Follow-up ultrasound study image shows more organization of the abscess, with an increasing cystic component (arrowhead). Aspiration was again performed, this time yielding $3 \mathrm{cc}$ of serous fluid. Biopsy was also done for this lesion this time, which revealed granulomatous mastitis.

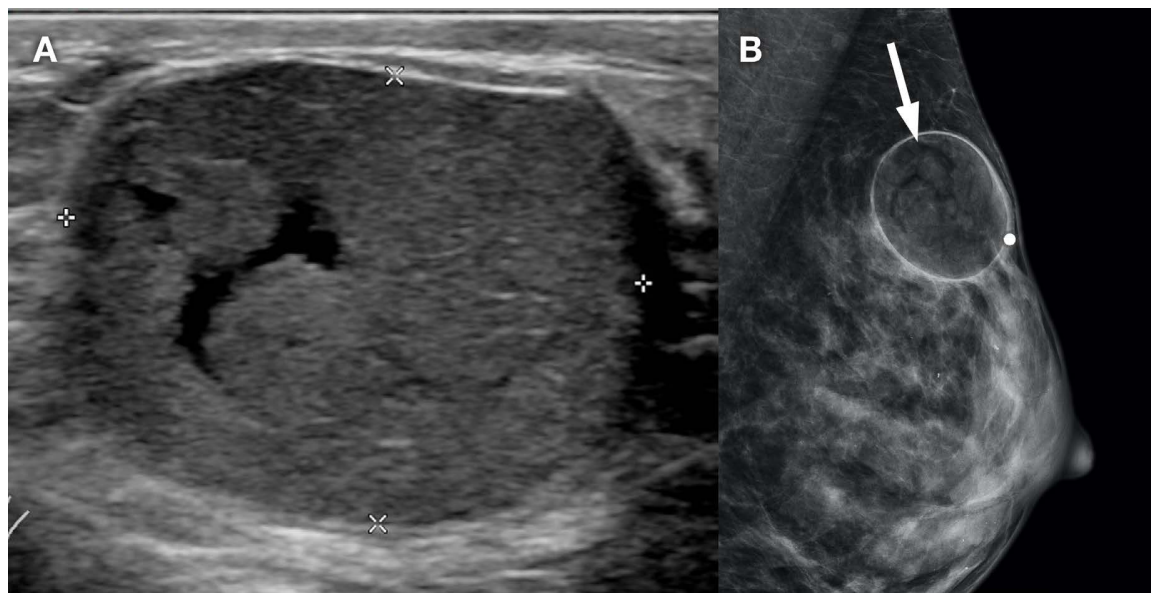

Figure 16. A 38-year-old female presented with a new, palpable lump in the left breast. The patient had stopped breastfeeding 5 months prior. (A) Sonographic images were initially taken which shows a type 4 CCBM, largely hypoechoic with scant cystic component; no internal vascularity was noted. Differential diagnoses of solid breast lesions (such as Phyllodes tumor) versus galactocoele were considered; (B) A supplementary one-view mammographic image shows the lesion in question to be of fat density (arrow), confirming a galactocoele. No intervention was indicated.

\section{Foreign body granuloma}

These can mimic soft tissue neoplasms on imaging, often causing diagnostic difficulty. Clinical history is therefore of major importance in considering reasonable differentials and management. There is typically an echogenic structure, and surrounding hypoechoic changes from granulomatous tissue response; in some cases, this can result in cystic foci (Figure 18). 


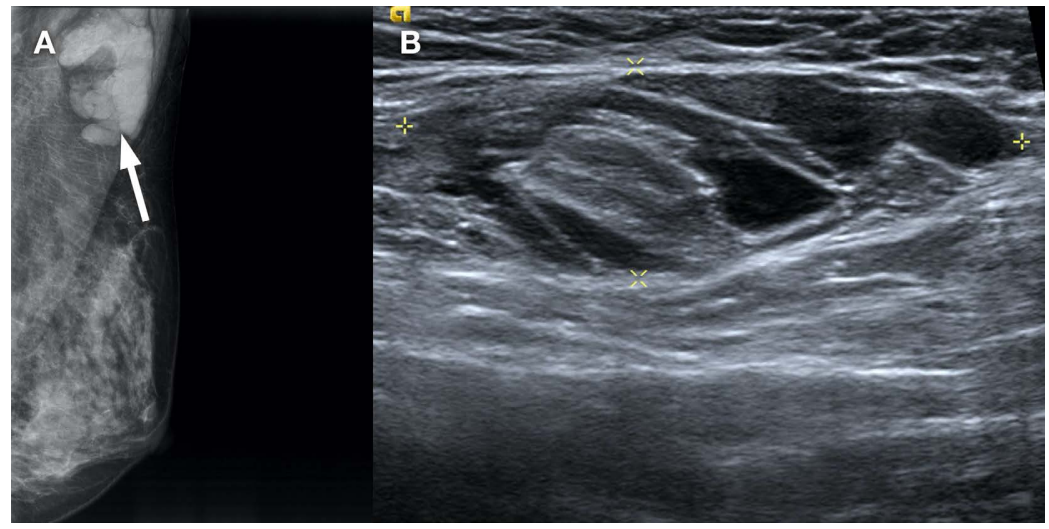

Figure 17. A 41-year-old female on screening mammogram was recalled for a left axillary isodense mass. (A) Mammogram shows an oval isodense opacity in the left axilla (arrow); (B) Sonographic image shows a type 4 CCBM, lobulated with a thick hypoechoic component, and small cystic components. After histology confirmed lymphangioma, the patient underwent curative excision of lesion, with no evidence of recurrence on 1-year followup.



Figure 18. A 35-year-old female with history of lumpectomy for DCIS a year prior underwent surveillance ultrasound. (A) and (B) Orthogonal sonographic images show a type 4 CCBM in the area of the scar (arrow)-largely hypoechoic and irregular with tiny eccentric cystic foci. Residual/recurrent tumor was considered in the differential diagnosis. Histology revealed a foreign body granuloma.

\section{Myxoid fibroadenoma}

Breast fibroadenomas feature proliferation of stromal and epithelial components of the TDLU. Myxoid fibroadenomas represent a histologic variant, where the stromal component is distinctly hypocellular with an abundant myxoid matrix. Classically they are well-defined, with rounded contours, generally hypoechoic (Figure 19); intralesional calcifications may be observed, as well as degenerative cystic foci.

Benign phyllodes tumor

Phyllodes tumors are rare fibroepithelial neoplasms, exhibiting rapid proliferation of epithelial and stromal cells. A benign mass may feature an inhomogeneous hypoechoic solid appearance, with well-defined, microlobulated margins and cleft-like cystic spaces internally (Figure 20) [14]. 


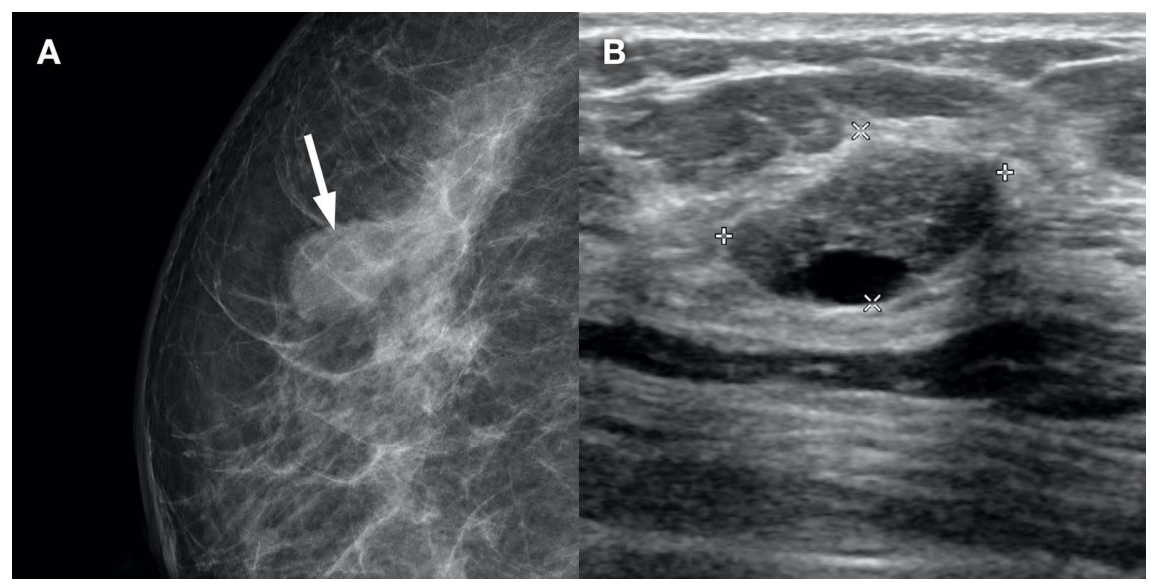

Figure 19. A 50-year-old patient with screen-detected right breast mass. (A) Mammogram shows a partially-circumscribed isodense mass in the right breast (arrow); (B) Corresponding sonographic image in the 10 o'clock location shows a well-circumscribed, oval hypoechoic mass with eccentric cystic component, consistent with a type 4 CCBM. Histology was reported as myxoid fibroadenoma.

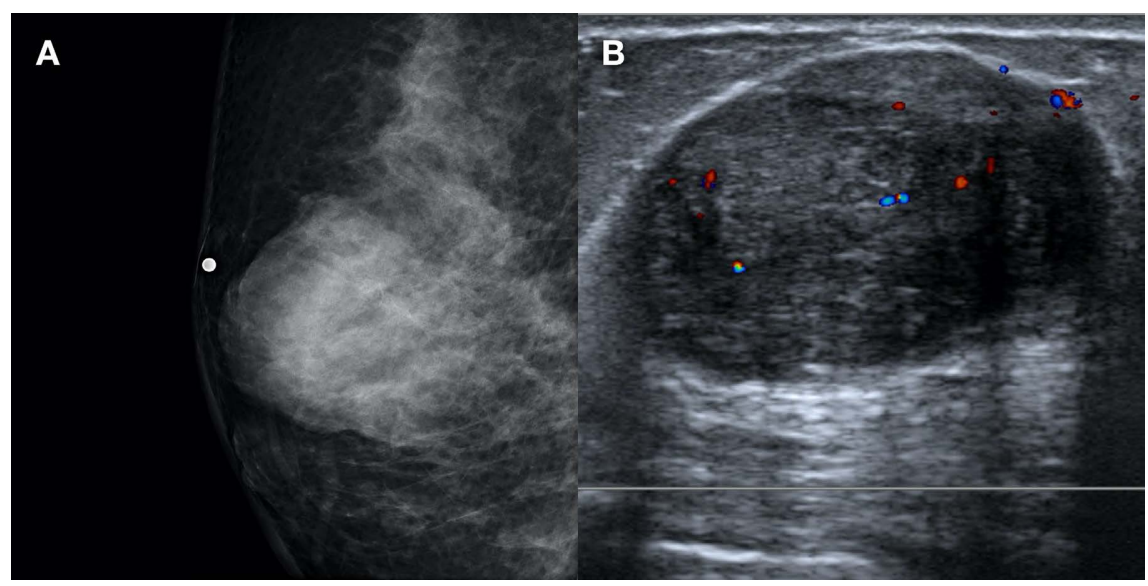

Figure 20. A 60-year-old female with palpable lump in right breast. (A) Mammogram shows a circumscribed, oval, high-density mass in the right breast; (B) Corresponding sonographic Doppler image shows a type 4 CCBM, predominantly solid with few cystic changes within and mild internal vascularity. Histology was reported as benign phyllodes.

Invasive ductal carcinoma (IDC)

The most common breast malignancy, comprising up to $80 \%$ of cases, this presents as a firm, palpable and painful mass, sometimes with skin thickening and erythema, nipple retraction and discharge. Originating in the duct and infiltrating its basement membrane, it grows and replaces the surrounding tissues indiscriminately. Suspicious features include the following: ill-defined/angular margins, spiculated pattern, anti-parallel configuration, with internal microcalcifications. Occasionally they are mixed solid-cystic, which confers a higher malignancy grade on histology [15] (Figure 21).

Invasive mucinous carcinoma

Accounting for $2 \%$ of breast malignancies, these are divided into pure and mixed mucinous subtypes. The former features a larger amount of mucin, and is 


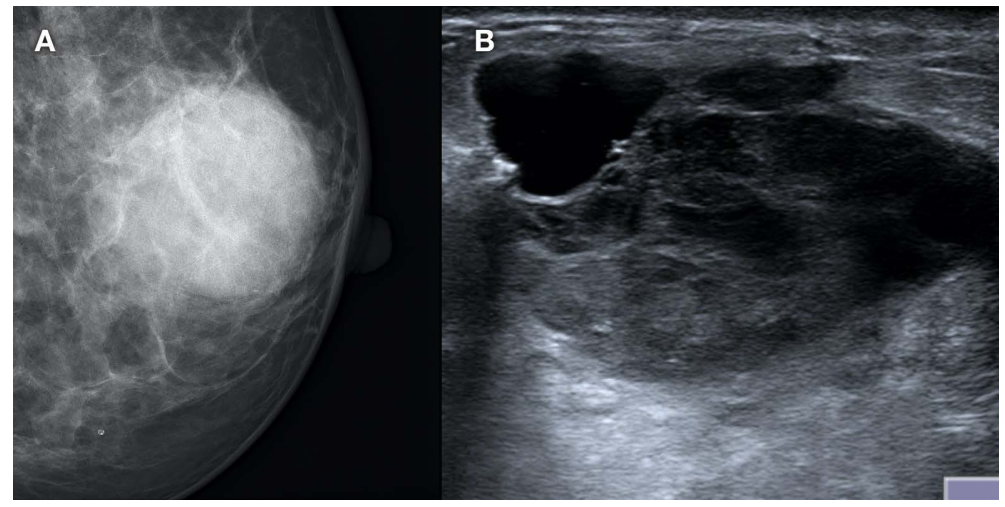

Figure 21. A 68-year-old female presented with a palpable mass in the left breast. (A) Mammographic spot CC image shows a partially-circumscribed high-density mass in the retroareolar region of the left breast; (B) Sonographic image shows a type 4 CCBM in the corresponding location, with an eccentric cystic component. Histology was reported as grade 3 invasive ductal carcinoma.

less aggressive with lower rates of axillary nodal metastases. They demonstrate mixed cystic and solid components, with microlobulated margins; posterior acoustic enhancement is common (Figure 22). The pure subtype tends to be more homogeneous, reflecting its less aggressive nature [16].

Ductal carcinoma in situ (DCIS)

Accounting for $15 \%-20 \%$ of breast malignancies, this frequently asymptomatic condition exhibits proliferation of malignant cells within ducts, without basement membrane invasion. If suspicious microcalcifications are detected on mammogram, features allowing for US-guided biopsy may be sought, which is generally preferred over stereotactic biopsy. Calcified DCIS may appear with echogenic foci located within a mass or a duct, sometimes distributed in a branch pattern. Non-calcified DCIS features rounded/irregular hypoechoic masses, with indistinct or microlobulated margins (Figure 23) [17].

Encysted papillary carcinoma

This rare subtype of papillary carcinoma comprises $1 \%-2 \%$ of breast malignancies. It usually presents post-menopausally as a slow-growing, palpable retroareolar mass with nipple discharge. A complex cystic mass is seen, sometimes with septations, and vascular solid papillary components projecting into the cyst lumen [18] (Figure 24).

Malignant phyllodes tumor

Imaging features favoring malignant phyllodes include large size (generally larger than $3-7 \mathrm{~cm}$ [14]), irregular margins with an echogenic rim, inhomogeneous hypoechoic echotexture and vascularized solid components (Figure 25). Cleft-like anechoic spaces within suggest focal necrosis or cystic degeneration.

Metaplastic breast carcinoma

This rare, heterogeneous group of malignancies accounts for $<1 \%$ of all mammary tumors. Histologically diverse, they are divided into subtypes exhibiting varying patterns of metaplasia and differentiation with epithelial and/or mesenchymal components. Patients present with rapidly growing large masses, with 


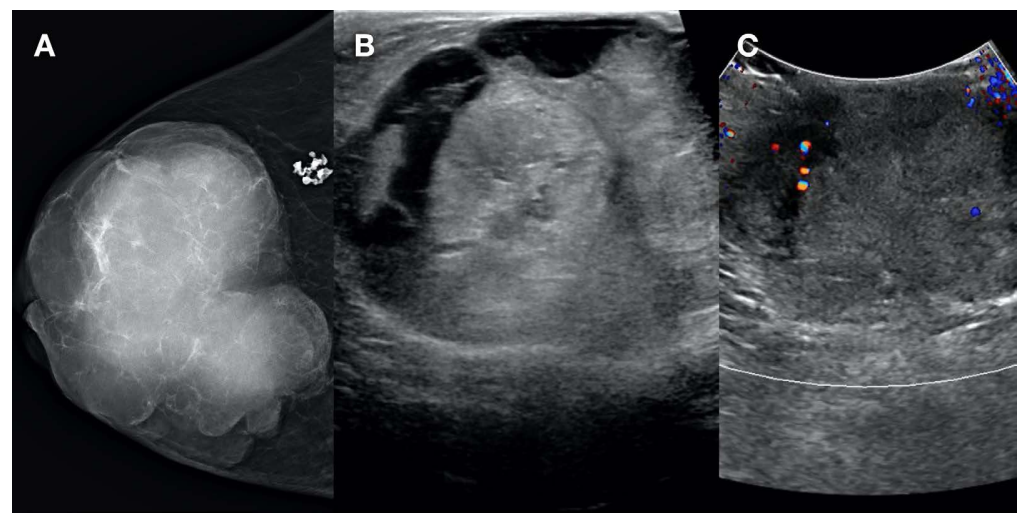

Figure 22. A 78-year-old female diagnosed with invasive mixed mucinous carcinoma of right breast. (A) Mammogram shows a massive, lobulated high-density mass, occupying most of the right breast; (B) Corresponding sonographic image shows a large type 4 CCBM; (C) Internal vascularity is demonstrated on color Doppler.



Figure 23. A 38-year-old female with family history of breast and ovarian cancer, on regular follow-up of breast nodules. (A) One of the circumscribed hypoechoic nodules in the 10 o'clock region shows small eccentric cystic changes, consistent with a type 4 CCBM; (B) Internal vascularity is also demonstrated in the solid component. Hence, biopsy was considered, which later revealed DCIS.



Figure 24. A 74-year-old female presented with a large palpable mass in the left breast. (A) Left mammogram shows an irregular dense mass; (B) Corresponding sonographic image shows a large, type 4 CCBM with irregular cystic foci noted within. Significant vascularity was noted in the solid component (not shown). Histology was reported as encysted papillary carcinoma. 
infrequent axillary nodal involvement. These are well-circumscribed, and may be rounded or microlobulated, exhibiting complex internal echogenicity, with anechoic components indicating necrosis or cystic degeneration (Figure 26 and Figure 27) [19].

\section{Angiosarcoma}

This exceedingly rare malignant tumor of endovascular origin accounts for about $0.04 \%$ of breast cancers. Primary and secondary angiosarcomas have been described, the former occurring sporadically from 20 - 40 years of age, and the latter in older patients after breast radiotherapy [20]. Sonographically it is non-specific, appearing circumscribed or ill-defined, with hypoechoic, hyperechoic, or heterogeneous echotexture and presence of vascularity (Figure 28). As existing post-therapy changes can complicate the findings, radiologists should be cognizant of prior history.

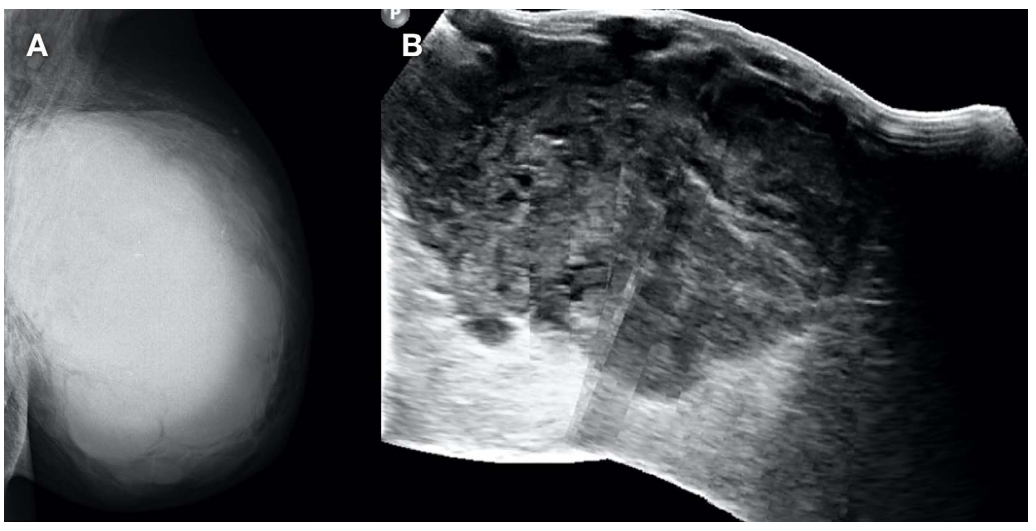

Figure 25. A 62-year-old female presented with a large mass occupying almost the entire left breast. (A) Mammogram shows a large, lobulated highly dense mass without suspicious microcalcifications; (B) Corresponding sonographic image shows a large type 4 CCBM, with microlobulated margins, heterogeneous echogenicity with tiny scattered cystic foci. Histology was reported as malignant phyllodes.

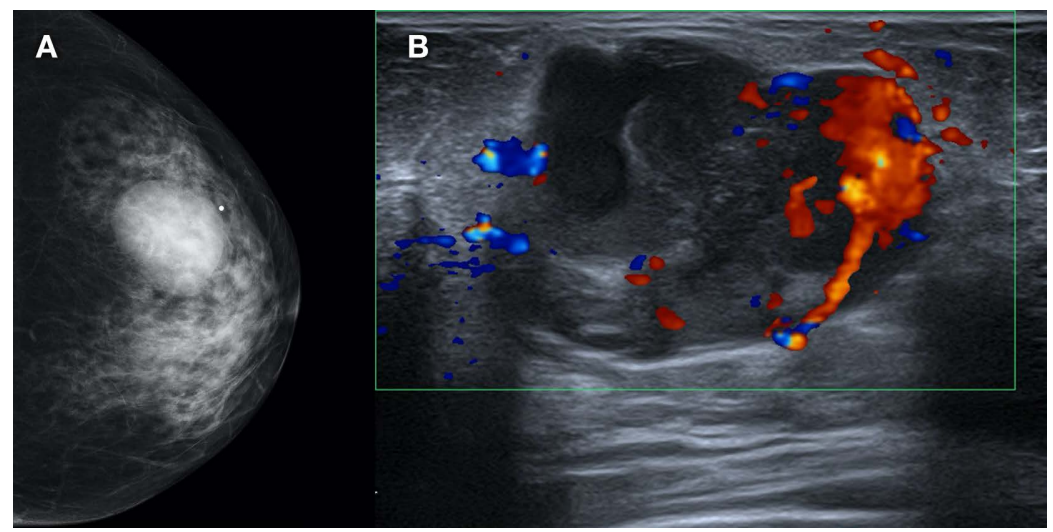

Figure 26. Metaplastic breast carcinoma, sarcomatoid carcinoma subtype, which features a mixed epithelial-mesenchymal pattern. A 61-year-old female presented with a palpable left breast lump. (A) Mammogram shows a lobulated high-density mass in the left upper outer quadrant; (B) Corresponding sonographic Doppler image shows a type 4 CCBM, showing a solid-cystic mass with significant internal vascularity and mobile echoes. 




Figure 27. Metaplastic breast carcinoma, spindle cell carcinoma subtype, which features a pure epithelial pattern. A 62-year-old female with previous history of right-sided wide excision and axillary clearance presented with a new, large right breast mass. (A) Sonographic image shows a type $4 \mathrm{CCBM}$ with superficial cystic components; (B) Internal vascularity is noted in the solid component on color Doppler.

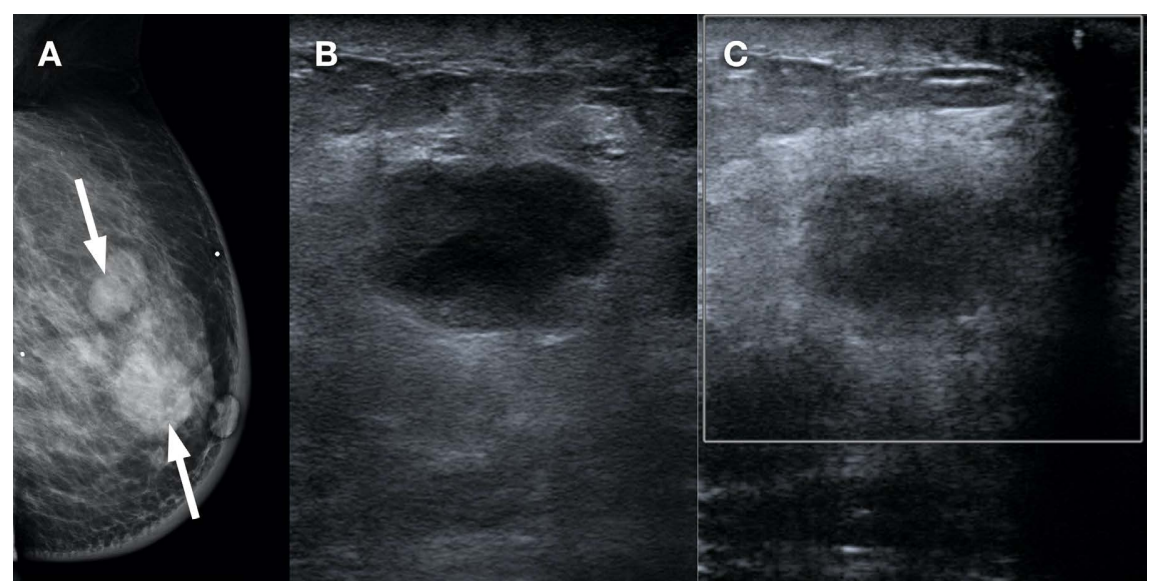

Figure 28. A 63-year-old female with previous lumpectomy and radiation therapy for left breast cancer, 10-year prior, presented with suspicion of recurrence. (A) Mammogram shows multiple new masses in the left breast (arrows), with overlying skin thickening and tissue oedema; (B) Sonographic image shows a type 4 CCBM in the 12 o'clock region corresponding to the mammographic findings; (C) In this case, the lesion does not show significant internal vascularity. Histology confirmed this lesion to be angiosarcoma.

\section{Conclusion}

We conclude by summarizing a few teaching points. First, CCBMs with a higher risk of malignancy $(20 \%-30 \%)$, are generally classified under the BI-RADS 4 category. Second, CCBMs have a wide range of differential diagnoses, with a variety of benign, borderline and malignant histologies. Classifying these lesions based on ultrasound morphology can be useful in assessing common imaging features, helping to draw attention to their differences and their clinical implications. Type 3 and type 4 CCBMs are more likely to be malignant compared to types 1 and 2. Third, biopsies should be done under ultrasound guidance to target the solid component and to avoid false negative results. Radiology pathology concordance 
is of utmost significance in these cases. Finally, clinical history and mammographic correlation may help to narrow the differential diagnoses and prevent unnecessary biopsies in certain CCBMs, such as post-operative seromas and galactoceles.

\section{Acknowledgements}

This paper was not supported by any financial grants or funding body.

\section{Conflicts of Interest}

No potential conflict of interest relevant to this article was reported.

\section{References}

[1] Doshi, D.J., March, D.E., Crisi, G.M. and Coughlin, B.F. (2007) Complex Cystic Breast Masses: Diagnostic Approach and Imaging-Pathologic Correlation. RadioGraphics, 27, S53-S64. https://doi.org/10.1148/rg.27si075508

[2] Berg, W.A., Campassi, C.I. and Ioffe, O.B. (2003) Cystic Lesions of the Breast: Sonographic-Pathologic Correlation. Radiology, 227, 183-191. https://doi.org/10.1148/radiol.2272020660

[3] Cho, S.H. and Park, S.H. (2013) Mimickers of Breast Malignancy on Breast Sonography. Journal of Ultrasound in Medicine, 32, 2029-2036. https://doi.org/10.7863/ultra.32.11.2029

[4] Solorzano, S., Mesurolle, B., Omeroglu, A., El Khoury, M., Kao, E., Aldis, A., et al. (2011) Flat Epithelial Atypia of the Breast: Pathological-Radiological Correlation. American Journal of Roentgenology, 197, 740-746. https://doi.org/10.2214/AJR.10.5265

[5] Gokhale, S. (2009) Ultrasound Characterization of Breast Masses. Indian Journal of Radiology and Imaging, 19, 242-247.

[6] Warner, J.K., Kumar, D. and Berg, W.A. (1998) Apocrine Metaplasia: Mammographic and Sonographic Appearances. American Journal of Roentgenology, 170, 1375-1379. https://doi.org/10.2214/ajr.170.5.9574619

[7] Sarakbi, W.A., Worku, D., Escobar, P.F. and Mokbel, K. (2006) Breast Papillomas: Current Management with a Focus on a New Diagnostic and Therapeutic Modality. International Seminars in Surgical Oncology, 3, Article No. 1. https://doi.org/10.1186/1477-7800-3-1

[8] Eiada, R., Chong, J., Kulkarni, S., Goldberg, F. and Muradali, D. (2012) Papillary Lesions of the Breast: MRI, Ultrasound, and Mammographic Appearances. American Journal of Roentgenology, 198, 264-271. https://doi.org/10.2214/AJR.11.7922

[9] Mesurolle, B., Perez, J.C.H., Azzumea, F., Lemercier, E., Xie, X., Aldis, A., et al. (2014) Atypical Ductal Hyperplasia Diagnosed at Sonographically Guided Core Needle Biopsy: Frequency, Final Surgical Outcome, and Factors Associated With Underestimation. American Journal of Roentgenology, 202, 1389-1394. https://doi.org/10.2214/AJR.13.10864

[10] Taboada, J.L., Stephens, T.W., Krishnamurthy, S., Brandt, K.R. and Whitman, G.J. (2009) The Many Faces of Fat Necrosis in the Breast. American Journal of Roentgenology, 192, 815-825. https://doi.org/10.2214/AJR.08.1250

[11] Lee, J.H., Kim, S.H., Kang, B.J., Lee, A.W. and Song, B.J. (2010) Ultrasonographic Features of Benign Adenomyoepithelioma of the Breast. Korean Journal of Radiol- 
ogy, 11, 522-527.

[12] Kim, M.J., Kim, E.K., Park, S.Y., Jung, H.K., Oh, K.K. and Seok, J.Y. (2006) Galactocoeles Mimicking Suspicious Solid Masses on Sonography. Journal of Ultrasound in Medicine, 25, 145-151. https://doi.org/10.7863/jum.2006.25.2.145

[13] Chung, S.Y., Oh, K.K. and Kim, D.J. (2003) Mammographic and Sonographic Findings of a Breast Cystic Lymphangioma. Journal of Ultrasound in Medicine, 22, 307-309. https://doi.org/10.7863/jum.2003.22.3.307

[14] Kalambo, M., Adrada, B.E., Adeyefa, M.M., Krishnamurthy, S., Hess, K., Carkaci, S., et al. (2018) Phyllodes Tumour of the Breast: Ultrasound-Pathology Correlation. American Journal of Roentgenology, 210, W173-W179.

https://doi.org/10.2214/AJR.17.18554

[15] Gupta, K., Kumaresan, M., Venkatesan, B., Chandra, T., Patil, A. and Menon, M. (2018) Sonographic Features of Invasive Ductal Breast Carcinomas Predictive of Malignancy Grade. Indian Journal of Radiology and Imaging, 28, 123-131.

https://doi.org/10.4103/ijri.IJRI $257 \quad 17$

[16] Lam, W.W.M., Chu, W.C.W., Tse, G.M. and Ma, T.K. (2004) Sonographic Appearance of Mucinous Carcinoma of the Breast. American Journal of Roentgenology, 182, 1069-1074. https://doi.org/10.2214/ajr.182.4.1821069

[17] Wang, L., Sullivan, M., Du, H., Feldman, M.I. and Mendelson, E.B. (2013) US Appearance of Ductal Carcinoma in Situ. RadioGraphics, 33, 213-228. https://doi.org/10.1148/rg.331125092

[18] Dogan, B.E., Whitman, G.J., Middleton, L.P. and Phelps, M. (2003) Intracystic Papillary Carcinoma of the Breast. American Journal of Roentgenology, 181, 186. https://doi.org/10.2214/ajr.181.1.1810186

[19] Günhan-Bilgen, I., Memiş, A., Ustün, E.E., Zekioglu, O. and Ozdemir, N. (2002) Metaplastic Carcinoma of the Breast: Clinical, Mammographic, and Sonographic Findings with Histopathologic Correlation. American Journal of Roentgenology, 178, 1421-1425. https://doi.org/10.2214/ajr.178.6.1781421

[20] Lim, R.F. and Goei, R. (2007) Angiosarcoma of the Breast. RadioGraphics, 27, S125S130. https://doi.org/10.1148/rg.27si075016 